Research Article

\title{
Combined effects of Hall and ion-slip current on MHD free convection flow in a vertical micro-channel
}

\author{
Basant K. Jha ${ }^{1} \cdot$ Peter B. Malgwi ${ }^{1}$ (D)
}

๑ Springer Nature Switzerland AG 2019

\begin{abstract}
Theoretic analysis on buoyancy-driven flow of conducting fluid in a vertical micro-channel taking into consideration the effect of Hall and ion-slip current is investigated. The micro-channel walls are assumed to be heated asymmetrically and flow is induced by buoyancy forces. The governing couple equations are solved exactly using the method of undetermined coefficient. Solutions are presented in dimensionless form. Effect of rarefaction parameter, Hartmann number, Hall current parameter and ion-slip parameter on fluid velocity, volume flow rate and skin friction along primary along primary and secondary flow directions are discussed.
\end{abstract}

Keywords Hall current · Ion-slip current · Micro-channel · Velocity slip · Temperature jump · Free convection

\section{List of symbols}

$H_{0} \quad$ Constant magnetic flux density (Tesla)

$g \quad$ Acceleration due to gravity $\left(\mathrm{m} \mathrm{s}^{-2}\right)$

$b \quad$ Channel width $(\mathrm{m})$

$C_{p}, C_{v}$ Specific heat at constant pressure and constant volume, respectively $\left(\mathrm{J} \mathrm{kg}^{-1} \mathrm{~K}^{-1}\right)$

$f_{\mathrm{t}}, f_{\mathrm{v}} \quad$ Thermal and tangential momentum accommodation coefficient, respectively (dimensionless variables)

$\beta_{\mathrm{t}} \quad$ Temperature jump (dimensionless variables)

$\beta_{\mathrm{v}} \quad$ Velocity slip (dimensionless variables)

$\lambda \quad$ Mean free path (m)

In Fluid-wall interaction parameter $\beta_{\mathrm{t}} / \beta_{\mathrm{v}}$ (dimensionless variables)

Kn Knudsen number $\lambda / b$ (dimensionless variables)

$\delta \quad$ Dimensionless volume flow rate

$M \quad$ Hartmann number (dimensionless)

$u^{\prime} \quad$ Dimensional velocity along the $x^{\prime}$-direction $\left(\mathrm{m} \mathrm{s}^{-1}\right)$

$w^{\prime} \quad$ Dimensional velocity along the $z^{\prime}$-direction $\left(\mathrm{m} \mathrm{s}^{-1}\right)$

$U_{0} \quad$ Characteristic velocity $\left(\mathrm{m} \mathrm{s}^{-1}\right)$

$u \quad$ Velocity along the $x$-direction (dimensionless)
W Velocity along the $z$-direction (dimensionless)

$Q \quad$ Complex velocity (dimensionless)

$\theta \quad$ Temperature (dimensionless)

$\omega_{\mathrm{e}} \quad$ Cyclotron frequency of electrons

$\omega_{\mathrm{i}} \quad$ Cyclotron frequency of ions

$\tau_{\mathrm{e}} \quad$ Electron collision time

$\tau_{\mathrm{i}} \quad$ Ion collision time

$\beta_{\mathrm{e}} \quad$ Hall parameter $\left(\omega_{\mathrm{e}} \tau_{\mathrm{e}}\right)$

$\beta_{\mathrm{i}} \quad$ Ion-slip parameter $\left(\omega_{\mathrm{i}} \tau_{\mathrm{i}}\right)$

$T \quad$ Temperature of the fluid (K)

$T_{1} \quad$ Temperature of the right wall (K)

$T_{2} \quad$ Temperature of the left wall (K)

\section{Greek letters}

$\beta \quad$ Coefficient of thermal expansion $\left(\mathrm{K}^{-1}\right)$

$\gamma_{\mathrm{s}} \quad$ Ratio of specific heat $C_{\mathrm{p}} / C_{\mathrm{v}}$

$\sigma \quad$ Electrical conductivity of the fluid

$\mu_{\mathrm{e}} \quad$ Magnetic permeability $\left(\mathrm{N} \mathrm{A}^{-2}\right)$

$\xi \quad$ Wall ambient temperature (dimensionless)

$\rho \quad$ Density $\left(\mathrm{kg} \mathrm{m}^{-3}\right)$

Peter B. Malgwi, bumalpeter@gmail.com; Basant K. Jha, basant777@yahoo.co.uk|'Department of Mathematics, Ahmadu Bello University, Zaria, Nigeria. 


\section{Introduction}

Studies on hydromagnetic free convection flow of viscous incompressible and electrically conducting fluids like ionized gases (plasmas), liquid metal (mercury or liquid sodium) [1], in micro-channels are of immense relevance owing to its many scientific and technological applications like microelectromechanical system (MEMS), nanoelectromechanical system (NEMS), micropumps, micro-heat sink, flow meters, drug delivery, material processing, cooling of microelectronic devices, liquid robotics and colloidal energetic systems $[2,3]$. In view of these applications, a lot of research have been conducted to improve the efficiency and performance of these devices. Some articles on hydromagnetic free convection flow under different physical situations include: Jha et al. [4], Jha and Aina [5-7], Avci and Aydin $[8,9]$, Bounomo and Manca [10], Hossain and Mandal [11], Ibrahim et al. [12], Eldabe et al. [13] and Gorla and Chamkha [14].

It is well known that subjecting the flow of electrically conducting fluid to a strong externally applied magnetic field induces many complex electromagnetic phenomena on flow characteristics such as Hall current, ion slip and Joule heating as stated by Cramer and Pai [15]. The Hall current becomes noticeable when an ionized gas with low density experiences the influence of a strong magnetic field (Sato [16]). Such types of flows are likely to find relevance in underground energy storage systems, spacecraft propulsion technology and in sensor technologies. Datta and Jana [17] presented an exact solution for Hall effect on hydromagnetic free convection flow of conducting fluid in a channel. They observed that shear stress at the hotter wall could be reduced with the increase in Hall parameter. In a related work, Datta and Jana [18] analyzed the effect of Hall current on mixed convection hydromagnetic flow of viscous incompressible conducting fluid in a vertical channel. They observed that Hall current strengthens both primary fluid velocity and secondary fluid velocity, whereas it decreases the rate of heat transfer at the walls. Mazumder et al. [19] investigated the influence of Hall current on MHD Ekman layer flow in a permeable channel. Guchhait et al. [20] presented an exact solution for time-dependent hydromagnetic Couette flow of conducting fluid in a horizontal permeable channel in the presence of Hall current. They observed that at large time, Hall parameter increases the primary component of fluid velocity whereas it decreases along the secondary direction. Recently, Jha et al. [21] examined the effect of Hall current on hydromagnetic flow in a microchannel in the presence of natural convection current.
They recorded an increase in volume flow rate with the increase in Hall parameter. Other notable articles on Hall current having different geometric conditions include: Singh [22], Seth and Ansari [23], Seth and Singh [24], Makinde et al. [25], Krishna and Jyothi [26], Tani [27], etc.

In all the aforementioned studies on hydromagnetic flow of conducting fluid with Hall effects, the influence of ion-slip current is neglected in Ohm's law. In general, the diffusion velocity of electron is very large in comparison with diffusion velocity of ions. However, inclusion of combined effect of Hall current and ion-slip current becomes essential when the diffusion velocity of electrons and ions is of same order of magnitude. Mittal et al. [28] analyzed the effect of Hall current and ion-slip current on hydromagnetic flow in a rectangular channel. Ram et al. [29] examined the influence of Hall and ion-slip current on buoyancy-driven flow of conducting fluid in a rotating system. Jha and Apere [30] investigated the effect of Hall and ion-slip currents on hydromagnetic flow of viscous incompressible fluid in a region between two permeable parallel plates in a rotating system due to impulsive motion of one of the porous plates. Naroua et al. [31] presented an analysis on time-dependent flow of partially ionized gas in the presence of Hall and ion-slip current in a rotating channel with heat source. Singh et al. [32] studied combined effect of Hall and ion-slip current on unsteady hydromagnetic Couette-Poiseuille flow in the presence of a moving magnetic field. They found out that both primary and secondary component of fluid velocity decreases with the increase in ion-slip parameter. Srinivasacharya and Shafeeurrahman [33] studied the combined effect of Hall and ion-slip current on hydromagnetic nano-fluid flow of viscous incompressible and electrically conducting fluid in an annulus in the presence of mixed convection current. They recorded a reduction in fluid temperature due to the presence of Hall and ion-slip current, while it increases fluid velocity and nanoparticle volume fraction. Some interesting works on the above subject include: Attia and Aboul-Hassan [34], Singh et al. [35], Makinde et al. [36], Ghosh et al. [37], Mollah et al. [38], etc.

The objective of the present work is to discuss the impact of Hall and ion-slip current on hydromagnetic natural convection flow of viscous incompressible and electrically conducting fluid in a vertical micro-channel. The micro-channel walls are assumed to be heated asymmetrically. The governing equations responsible for the present mathematical are solved exactly using the method of undetermined coefficient under relevant boundary conditions and presented in dimensionless form. Effect of pertinent flow parameters is portrayed using line graphs and tables. In fact the present work is the generalization of Jha et al. [21] by incorporating ion-slip current. 


\section{Mathematical analysis}

Flow description of the present problem is shown in Fig. 1. Consider the steady, fully developed flow of viscous incompressible and electrically conducting fluid in a region between two vertical micro-channel walls situated at $y^{\prime}=0$ and $y^{\prime}=b$, respectively. The two walls are assumed to be heated asymmetrically with temperature $T_{1}$ at the right micro-channel wall and $T_{2}$ at the left wall. It is assumed that the temperature at the left wall $y^{\prime}=0$ is smaller than the temperature at the right wall $y^{\prime}=b$, i.e., $T_{1}>T_{2}$ giving rise to free convection current. A constant magnetic field is imposed perpendicular to the conducting fluid flow, along the $y^{\prime}$-axis. By assuming a negligibly small value for magnetic Reynolds number $\left(R_{m}=\sigma \mu_{\mathrm{e}} U_{0} b\right)$, the induced magnetic field is neglected. The effects of the applied magnetic field are assumed to be strong enough to induce Hall and ion-slip current in a direction perpendicular to both the electric and magnetic field. Hence, the velocity field and magnetic field vectors are of the form $\vec{V}=\left(u^{\prime}, 0, w^{\prime}\right)$ and $\vec{H}=\left(0, H_{0}, 0\right)$. Recently, Jha et al. [21] discussed Hall effect on MHD natural convection flow in a vertical micro-channel. Following, Jha et al. [21] and incorporating ion-slip current, the dimensional governing equation describing the above physical situation can be written as:

$\frac{\mathrm{d}^{2} u^{\prime}}{\mathrm{d} y^{\prime 2}}-\frac{\sigma H_{0}^{2} \mu_{\mathrm{e}}^{2}}{\left(\alpha_{\mathrm{e}}^{2}+\beta_{\mathrm{e}}^{2}\right) \rho}\left(\alpha_{\mathrm{e}} u^{\prime}+\beta_{\mathrm{e}} w^{\prime}\right)+g \beta\left(T^{\prime}-T_{0}\right)=0$

$\frac{\mathrm{d}^{2} w^{\prime}}{\mathrm{d} y^{\prime 2}}+\frac{\sigma H_{0}^{2} \mu_{\mathrm{e}}^{2}}{\left(\alpha_{\mathrm{e}}^{2}+\beta_{\mathrm{e}}^{2}\right) \rho}\left(\beta_{\mathrm{e}} u^{\prime}-\alpha_{\mathrm{e}} w^{\prime}\right)=0$

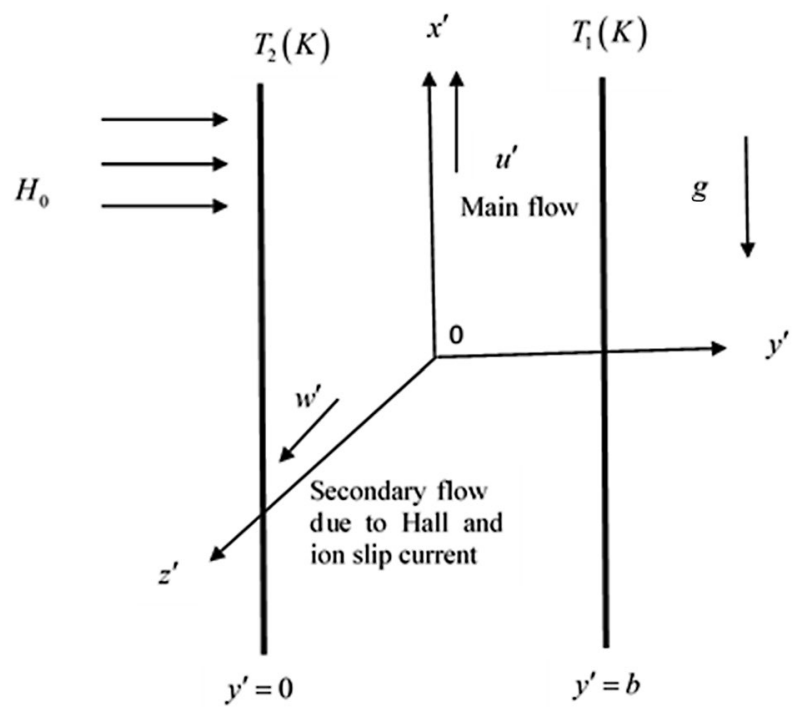

$\frac{d^{2} T^{\prime}}{d y^{\prime 2}}=0$

With dimensional momentum and thermal boundary conditions:

$u^{\prime}\left(y^{\prime}=0\right)=\left.\frac{2-f_{\mathrm{v}}}{f_{\mathrm{v}}} \lambda \frac{\mathrm{d} u^{\prime}}{\mathrm{d} y^{\prime}}\right|_{y^{\prime}=0}, w^{\prime}\left(y^{\prime}=0\right)=\left.\frac{2-f_{\mathrm{v}}}{f_{\mathrm{v}}} \lambda \frac{\mathrm{d} w^{\prime}}{\mathrm{d} y^{\prime}}\right|_{y^{\prime}=0}$,

$u^{\prime}\left(y^{\prime}=b\right)=-\left.\frac{2-f_{\mathrm{v}}}{f_{\mathrm{v}}} \lambda \frac{\mathrm{d} u^{\prime}}{\mathrm{d} y^{\prime}}\right|_{y^{\prime}=b}, w^{\prime}\left(y^{\prime}=b\right)=-\left.\frac{2-f_{\mathrm{v}}}{f_{\mathrm{v}}} \lambda \frac{\mathrm{d} w^{\prime}}{\mathrm{d} y^{\prime}}\right|_{y^{\prime}=b}$

$T^{\prime}\left(y^{\prime}=0\right)=T_{2}+\left.\frac{2-f_{\mathrm{t}}}{f_{\mathrm{t}}} \frac{2 \gamma_{s}}{\gamma_{s}+1} \frac{\lambda}{\operatorname{Pr}} \frac{\mathrm{d} T^{\prime}}{\mathrm{d} y^{\prime}}\right|_{y^{\prime}=0}$,

$T^{\prime}\left(y^{\prime}=b\right)=T_{1}-\left.\frac{2-f_{\mathrm{t}}}{f_{\mathrm{t}}} \frac{2 \gamma_{\mathrm{s}}}{\gamma_{\mathrm{s}}+1} \frac{\lambda}{\operatorname{Pr}} \frac{\mathrm{d} T^{\prime}}{\mathrm{d} y^{\prime}}\right|_{y^{\prime}=b}$.

Employing the dimensionless variables and parameters:

$y=\frac{y^{\prime}}{b}, M^{2}=\frac{\sigma \mu_{\mathrm{e}}^{2} H_{0}^{2} b^{2}}{\rho v},(u, w)=\frac{\left(u^{\prime}, w^{\prime}\right)}{U_{0}}, U_{0}=\frac{g \beta\left(T_{1}-T_{0}\right) b^{2}}{v}$,

$\operatorname{Pr}=\frac{v}{\alpha}, \theta=\frac{T^{\prime}-T_{0}}{T_{1}-T_{0}}$

Equations (1)-(3) can be rewritten in dimensionless form as:

$\frac{\mathrm{d}^{2} u}{\mathrm{~d} y^{2}}-\frac{M^{2}}{\left(\alpha_{\mathrm{e}}^{2}+\beta_{\mathrm{e}}^{2}\right)}\left(\alpha_{\mathrm{e}} u+\beta_{\mathrm{e}} w\right)+\theta=0$

$\frac{\mathrm{d}^{2} w}{\mathrm{~d} y^{2}}+\frac{M^{2}}{\left(\alpha_{\mathrm{e}}^{2}+\beta_{\mathrm{e}}^{2}\right)}\left(\beta_{\mathrm{e}} u-\alpha_{\mathrm{e}} w\right)=0$

$\frac{d^{2} \theta}{d y^{2}}=0$

With dimensionless momentum and thermal boundary conditions:

$u(y=0)=\left.\beta_{\mathrm{v}} K n \frac{\mathrm{d} u}{\mathrm{~d} y}\right|_{y=0}, w(y=0)=\left.\beta_{\mathrm{v}} K n \frac{\mathrm{d} w}{\mathrm{~d} y}\right|_{y=0}$,

$u(y=1)=-\left.\beta_{\mathrm{v}} K n \frac{\mathrm{d} u}{\mathrm{~d} y}\right|_{y=1}, w(y=1)=-\left.\beta_{\mathrm{v}} K n \frac{\mathrm{d} w}{\mathrm{~d} y}\right|_{y=1}$.

$\theta(y=0)=\xi+\left.\beta_{\mathrm{v}} K n \ln \frac{\mathrm{d} \theta}{\mathrm{d} y}\right|_{y=0}, \theta(y=1)=1-\left.\beta_{\mathrm{v}} K n \ln \frac{\mathrm{d} \theta}{\mathrm{d} y}\right|_{y=1}$.

where

$\beta_{\mathrm{v}}=\frac{2-f_{\mathrm{v}}}{f_{\mathrm{v}}}, \beta_{\mathrm{t}}=\frac{2-f_{\mathrm{t}}}{f_{\mathrm{t}}} \frac{2 \gamma_{\mathrm{s}}}{\gamma_{\mathrm{s}}+1} \frac{1}{\operatorname{Pr}}, K n=\frac{\lambda}{b}, \ln =\frac{\beta_{\mathrm{t}}}{\beta_{\mathrm{v}}}, \xi=\frac{T_{2}-T_{0}}{T_{1}-T_{0}}$.

Fig. 1 Flow description 
are, respectively, the velocity slip, temperature jump, Knudsen number, fluid wall interaction parameter and wall ambient temperature difference ratio as used in most microfluidic system. As defined in Eckert and Drake [39] and Goniak and Duffa [40], values for velocity slip and temperature jump at the micro-surfaces are $\beta_{v}=1.000$ and $\beta_{\mathrm{t}}=1.667$ corresponding to $\operatorname{Pr}=0.71, \gamma_{s}=1.4, f_{t}=f_{v}=1$.

By defining $F=u+i w$, Eqs. (6)-(7) and boundary conditions (9) can be combined as:

$\frac{d^{2} F}{d y^{2}}-M_{1}^{2} F+\theta=0$

where $M_{1}^{2}=\frac{M^{2}}{\alpha_{\mathrm{e}}+i \beta_{\mathrm{e}}}$ and $\alpha_{\mathrm{e}}=1+\beta_{\mathrm{e}} \beta_{\mathrm{i}}$

$\begin{array}{ll}F(0)=\left.\beta_{\mathrm{v}} K n \frac{\mathrm{d} F}{\mathrm{~d} y}\right|_{y=0} & F(1)=-\left.\beta_{\mathrm{v}} K n \frac{\mathrm{d} F}{\mathrm{~d} y}\right|_{y=1} \\ \theta(0)=\xi+\left.\beta_{\mathrm{v}} K n \ln \frac{\mathrm{d} \theta}{\mathrm{dy}}\right|_{y=0} \theta(1)=1-\left.\beta_{\mathrm{v}} K n \ln \frac{\mathrm{d} \theta}{\mathrm{d} y}\right|_{y=1}\end{array}$

Using the method of undetermined coefficient, solution of Eqs. (8) and (11) subject to the boundary conditions (12) gives:

$\theta(y)=C_{1}+C_{2} y$

$F(y)=C_{3} \cosh \left(M_{1} y\right)+C_{4} \sinh \left(M_{1} y\right)+\frac{1}{M_{1}^{2}}\left(C_{1}+C_{2} y\right)$

where

$C_{1}=\xi+\frac{\beta_{v} K n(1-\xi)}{1+2 \beta_{\mathrm{v}} K n \ln }, C_{2}=\frac{1-\xi}{1+2 \beta_{\mathrm{v}} K n \ln }$

$C_{3}=\frac{k_{1} k_{5}+k_{2} k_{4}}{k_{4}+k_{1} k_{3}}, \quad C_{4}=\frac{k_{5}-k_{2} k_{3}}{k_{4}+k_{1} k_{3}}$

$k_{1} \ldots k_{5}$ are constants defined in "Appendix."

\subsection{Special cases}

In the absence of ion-slip current, the present model reduces to the problem investigated by Jha et al. [21]. Also, in the absence of transversely applied magnetic field, the present work reduces to Chen and Weng [41].

Two important micro-channel flow characteristics are the volume flow rate and skin friction on the micro-channel surfaces. To obtain these features, we proceed as:

\subsection{Volume flow rate and skin friction}

Due to the combines effects of the Hall currents and ion slip, the present analysis considers the dimensionless volume flow rate in both the primary and secondary flow directions, given by;

$\delta=Q_{x}+i Q_{z}=\int_{0}^{1} F(y) d y$ where $Q_{x}$ and $Q_{z}$ represents the volume flow rate along the primary and secondary flow directions.

Substituting Eq. (14) into Eq. (15) gives:

$\delta=\frac{C_{3}}{M_{1}}\left(\sinh \left(M_{1}\right)\right)+\frac{C_{4}}{M_{1}}\left(\cosh \left(M_{1}\right)-1\right)+\frac{1}{M_{1}^{2}}\left(C_{1}+\frac{C_{2}}{2}\right)$

Similarly, the skin friction at the micro-channel walls for both primary and secondary flows situated on the walls $y=0$ and $y=1$ is obtained as:

$\tau_{0}=\tau_{x 0}+i \tau_{z 0}=\left.\frac{d F}{d y}\right|_{y=0}=M_{1} C_{4}+\frac{C_{2}}{M_{1}^{2}}$

$\tau_{1}=\tau_{x 1}+i \tau_{z 1}=\left.\frac{\mathrm{d} F}{\mathrm{~d} y}\right|_{y=1}=\frac{C_{2}}{M_{1}^{2}}-M_{1}\left(C_{3} \sinh \left(M_{1}\right)+C_{4} \cosh \left(M_{1}\right)\right)$

where $\tau_{x 0}, \tau_{z 0}$ and $\tau_{x 1}, \tau_{z 1}$ represent the skin friction along the primary and secondary flow directions at the microchannel walls $y=0$ and $y=1$, respectively.

\section{Results and discussion}

Using expression for fluid velocity, volume flow rate and skin friction obtained in the previous section, a MATLAB code is prepared to investigate effect of pertinent governing parameters on flow formation within the microchannel. Unless otherwise indicated, range of values used include: $5 \leq M \leq 9,0.01 \leq \beta_{\mathrm{e}} \leq 0.10,1 \leq \beta_{\mathrm{i}} \leq 9$ and $0.01 \leq \beta_{\mathrm{v}} K n \leq 0.10$ with fixed values $M=5, \beta_{\mathrm{i}}=3$, $\beta_{\mathrm{e}}=0.10, \mathrm{In}=1.667$ and $\beta_{\mathrm{v}} K n=0.05$ all arbitrarily chosen to investigate their effect on flow field as used in Jha et al. [21], Mollah et al. [38] and Chen and Weng [41]. The present analysis is carried out for three important cases of the wall ambient temperature difference ratio $\xi: \xi=1$ (symmetric heating) means the physical condition when the two micro-channel walls are heated symmetrically at the same constant temperature, $\xi=0$ (asymmetric heating) means the condition when one of the micro-channel wall is heated and the other not heated, $\xi=-1$ (asymmetric heating) means the physical condition when one of the walls is heated and the other is cooled at different ambient temperatures as defined in Weng and Chen [42].

Expression for energy equation in the micro-channel as given in Eq. (8) and solution in Eq. (13) is the same with those of Chen and Weng [40]. Detailed analysis on effect of rarefaction parameter $\left(\beta_{\mathrm{v}} K n\right)$, fluid wall interaction parameter (In) and wall ambient temperature difference ratio $(\xi)$ on fluid temperature and rate of heat transfer is discussed 
in their work. The main focus of this paper is to analyze the hydrodynamics of the flow in presence of Hall and ion-slip currents.

Figure $2 a$, b illustrates the effect of rarefaction parameter $\left(\beta_{\mathrm{v}} K n\right)$ on fluid velocity profile along primary and secondary flow direction in symmetrically $(\xi=1)$ or asymmetrically $(\xi=0,-1)$ heated micro-channel in the presence of Hall and ion-slip current. As expected, both primary and secondary fluid velocities are observed to be directly proportional to increase in $\beta_{\mathrm{v}} K n$ for $\xi=1$ and $\xi=0$ heating conditions yielding an increase in velocity profiles. This is due to the fact that the retarding effect of the boundary walls reduces with an increase in $\beta_{v} K n$. Figure $3(a, b)$ displays how the Hall current parameter $\left(\beta_{\mathrm{e}}\right)$ influences fluid

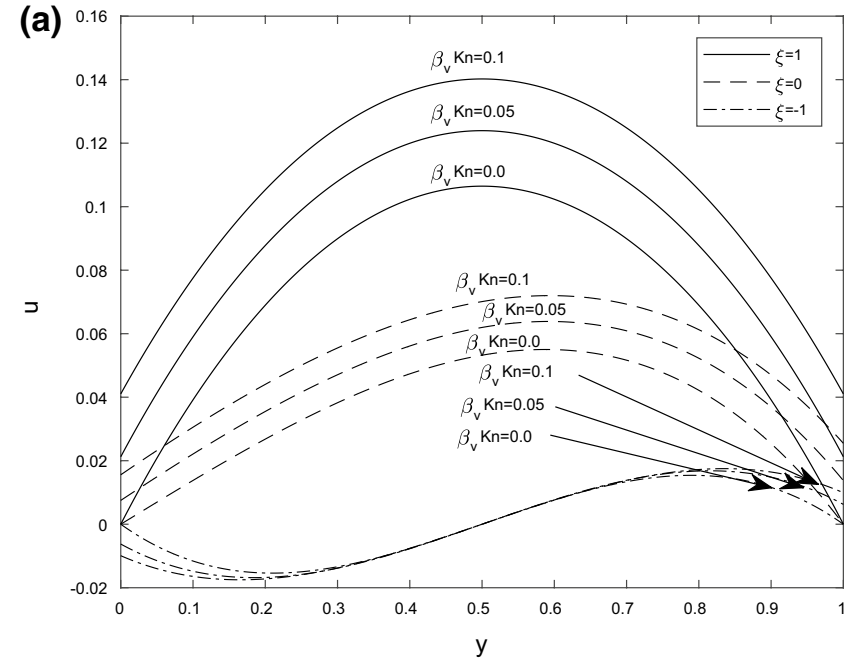

velocity distribution in the presence of ion-slip current parameter for different micro-channel heating conditions $(\xi)$. From these figures, we conclude that the impact of Hall parameter on primary velocity is almost negligible while it increases the secondary velocity for considered range of numerical values.

The effect of ion-slip $\left(\beta_{i}\right)$ parameter as well as wall ambient temperature difference ratio $(\xi)$ on fluid velocity is displayed in Fig. 4a, b for fixed values of Hall current parameter, Hartmann number, and rarefaction parameter. From the figure, it is interesting to observe that when the microchannel walls are heated either symmetrically $(\xi=1)$ or asymmetrically $(\xi=0)$, ion-slip parameter augment flow formation along the primary direction causes an increase (b)

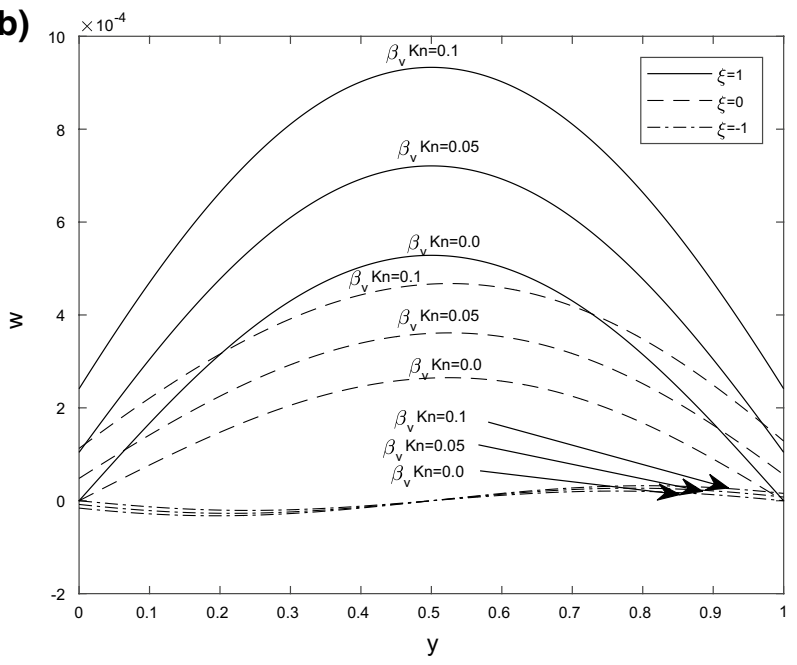

Fig. 2 Effect of rarefaction parameter $\left(\beta_{\mathrm{v}} K n\right)$ on primary and secondary velocity profiles for $\beta_{\mathrm{e}}=0.10, \beta_{\mathrm{i}}=3.0, \ln =1.667$ and $M=5.0$

(a)

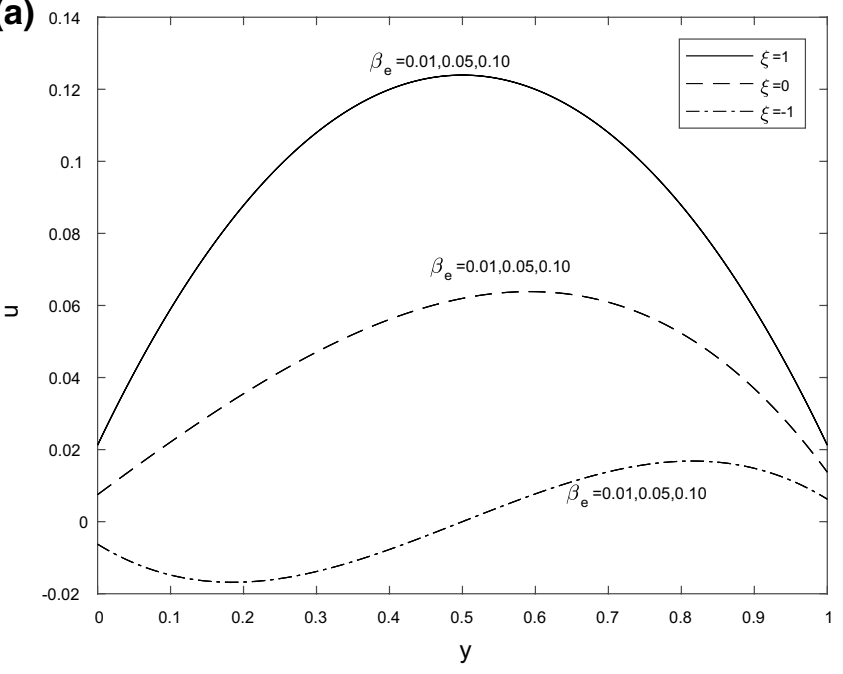

(b)

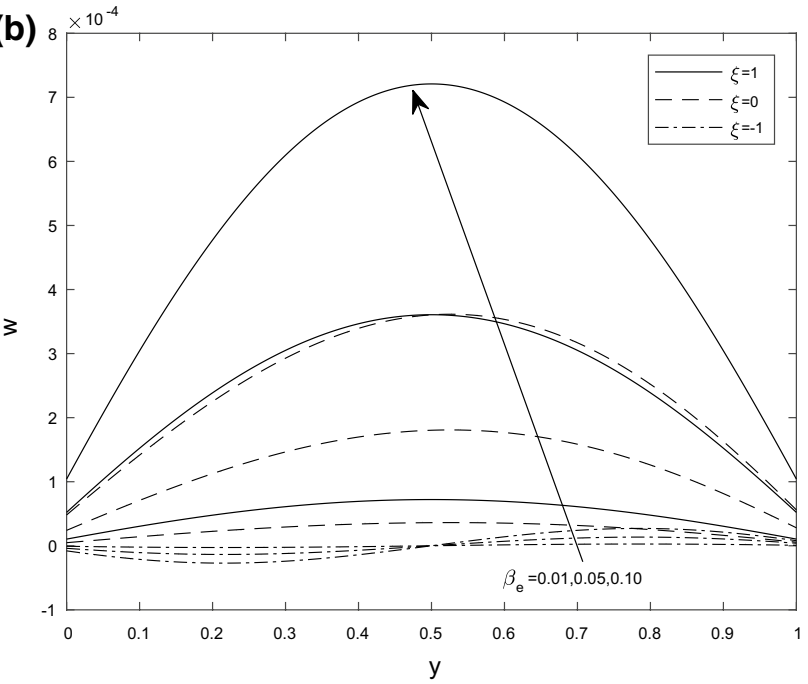

Fig. 3 Effect of Hall current parameter $\left(\beta_{\mathrm{e}}\right)$ on primary and secondary velocity profiles for $\beta_{\mathrm{v}} K n=0.05, \beta_{\mathrm{i}}=3.0$, In $=1.667$ and $M=5.0$ 

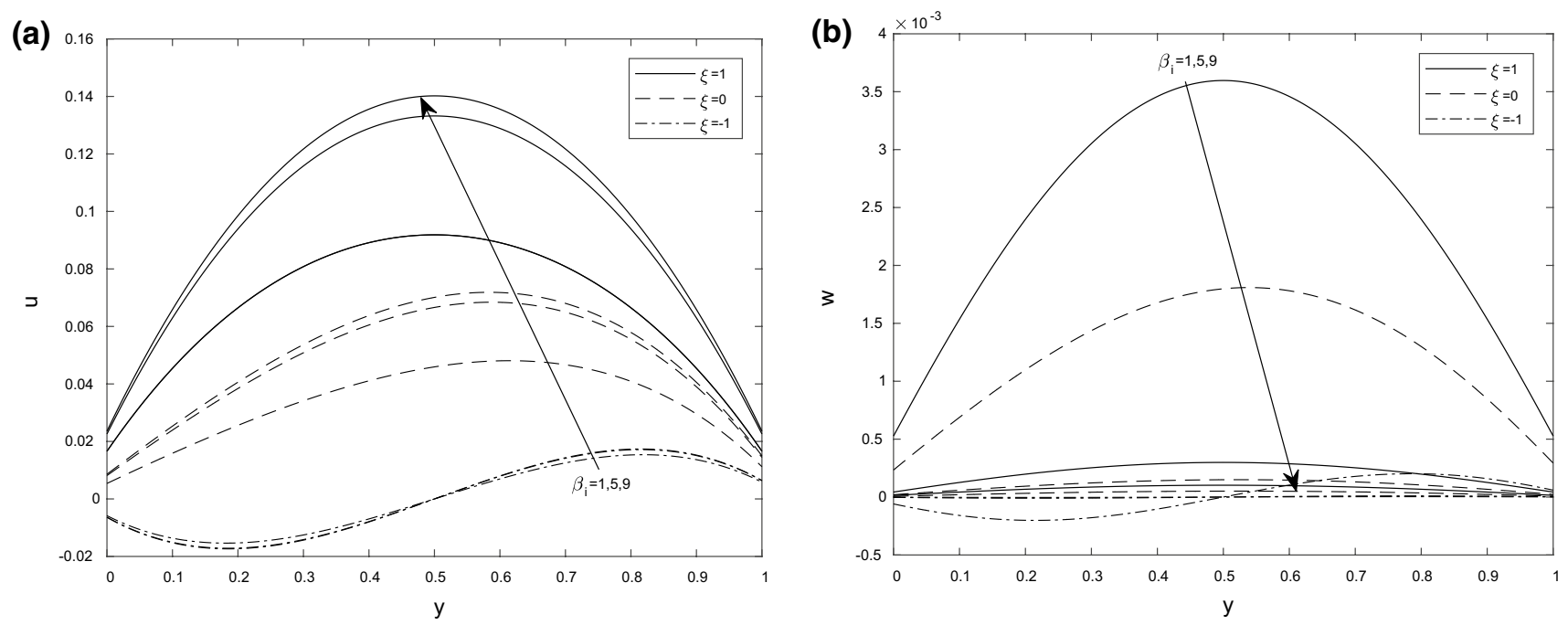

Fig. 4 Effect of ion-slip parameter $\left(\beta_{\mathrm{i}}\right)$ on primary and secondary velocity profiles for $\beta_{\mathrm{e}}=0.10, \beta_{\mathrm{v}} K n=0.05, \ln =1.667$ and $M=5.0$

in fluid velocity profile whereas it displays a dual character for asymmetric heating $(\xi=-1)$. The reverse of this phenomenon is evident along the secondary flow direction (Fig. 4b). Figure 5 illustrates the effect of Hartmann number $(M)$ in the presence of Hall $\left(\beta_{\mathrm{e}}\right)$ and ion-slip current $\left(\beta_{\mathrm{i}}\right)$ on fluid velocity for different values of $\xi$. The result indicates that for symmetric $(\xi=1)$ and asymmetric heating $(\xi=0,-1)$, the primary fluid velocity is lowered with the increase in Hartmann number all through the flow domain. This result is just contrast for secondary velocity.

Figures 6,7 and 8 examine the effect of Hartmann number $(M)$, Hall parameter $\left(\beta_{\mathrm{e}}\right)$, and ion-slip parameter $\left(\beta_{\mathrm{i}}\right)$ on volume flow rate along primary and secondary flow directions $\left(Q_{x}, Q_{z}\right)$ for different wall heating conditions $(\xi)$. The combined effect of Hall current parameter $\left(\beta_{\mathrm{e}}\right)$ and rarefaction parameter $\left(\beta_{\mathrm{v}} K n\right)$ on volume flow rate is portrayed in Fig. 6 for both symmetric $(\xi=1)$ and asymmetric $(\xi=0,-1)$ wall heating conditions. The figure reveals how the wall ambient temperature affects the influence of the Hartmann number on volume flow rate in the presence of Hall $\left(\beta_{\mathrm{e}}\right)$ and ion-slip current $\left(\beta_{\mathrm{i}}\right)$. It is evident from Fig. $6 \mathrm{a}$ that for $\xi=1$ and $\xi=0$, volume flow decreases along the primary flow direction with the increase in Hartmann number whereas it increases with the increase in rarefaction parameter $\left(\beta_{\mathrm{v}} K n\right)$. Interestingly, it is evident that for $\xi=0$, simultaneously increasing the Hartmann number $(M)$ and rarefaction parameter $\left(\beta_{v} K n\right)$ causes an enhancement in volume flow rate along secondary flow direction $\left(Q_{z}\right)$. In both flow directions, however, it is observed that

(b)

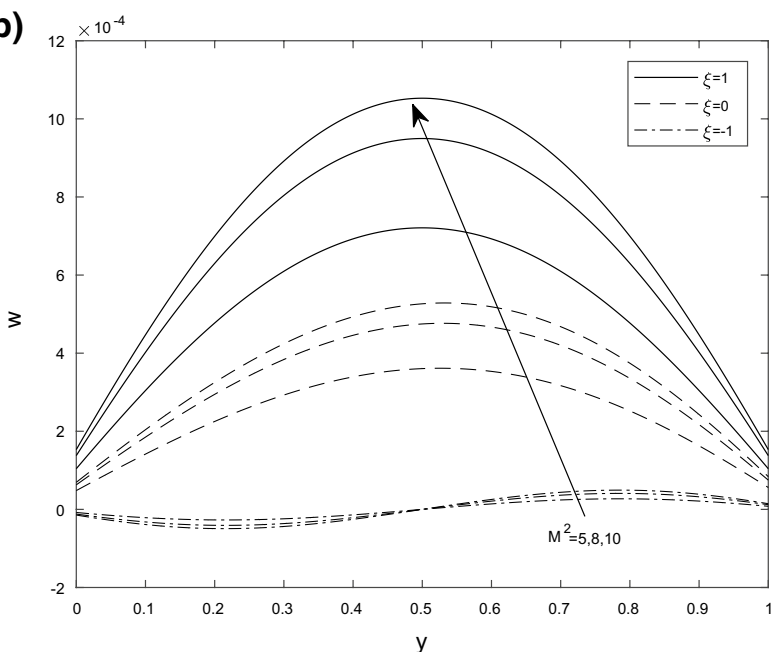

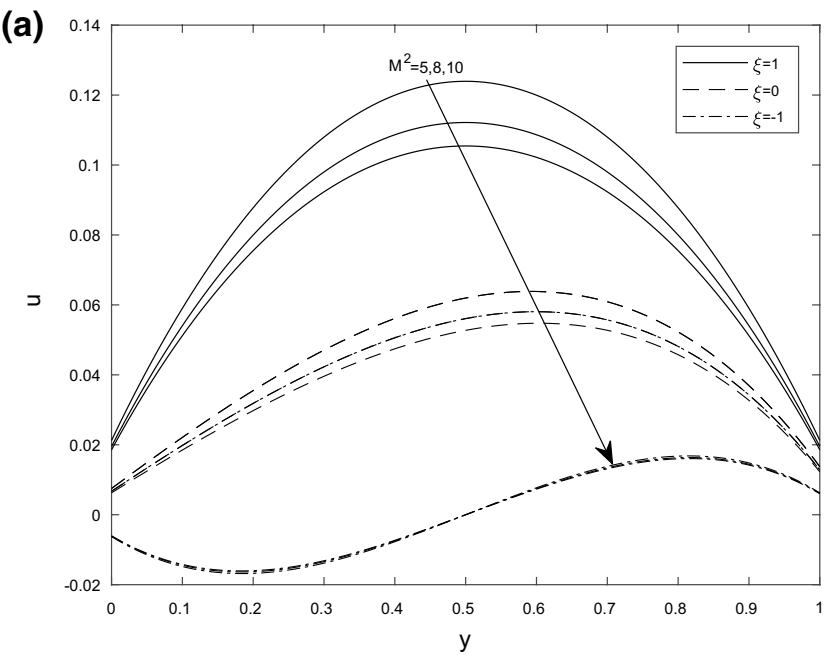

Fig. 5 Effect of Hartmann number $(M)$ on primary and secondary velocity profiles for $\beta_{\mathrm{e}}=0.10, \beta_{\mathrm{i}}=3.0, \ln =1.667$ and $\beta_{\mathrm{v}} \mathrm{Kn}=0.05$ 

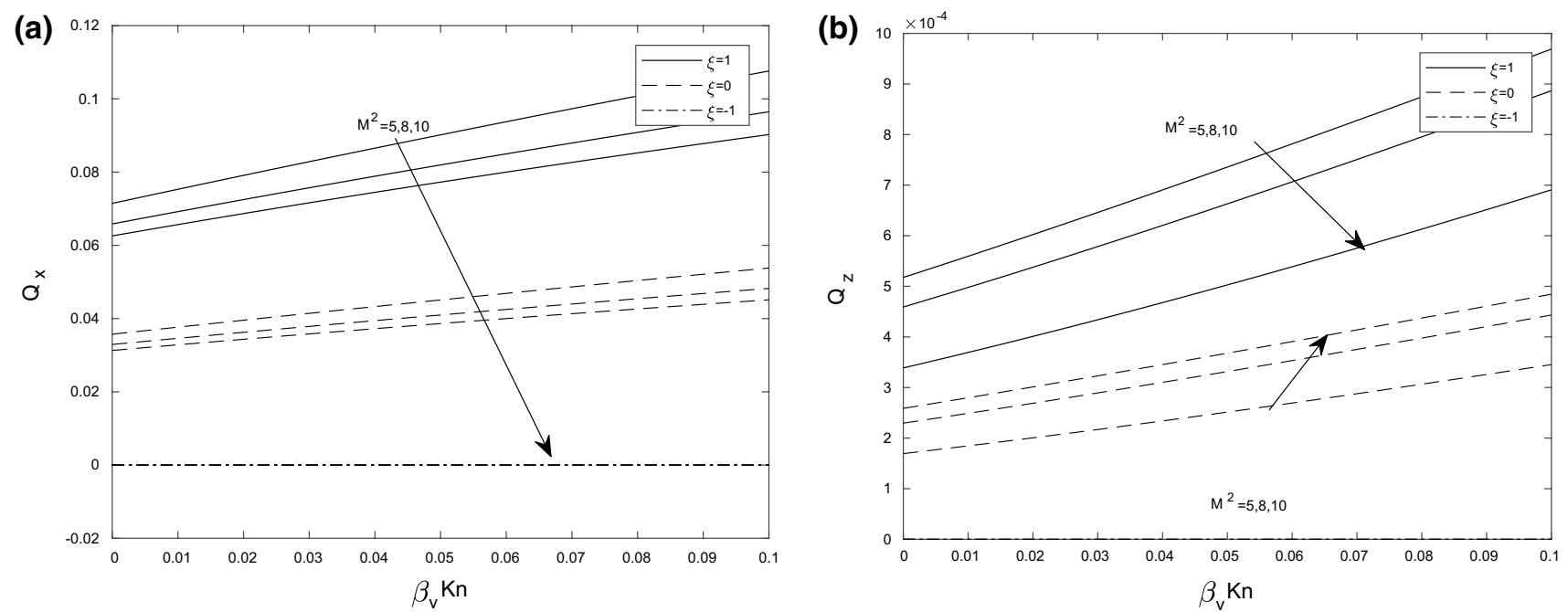

Fig. 6 Effect of Hartmann number $(M)$ on primary and secondary volume flow rate for $\beta_{\mathrm{e}}=0.10, \beta_{\mathrm{i}}=3.0$ and $\ln =1.667$

(a)

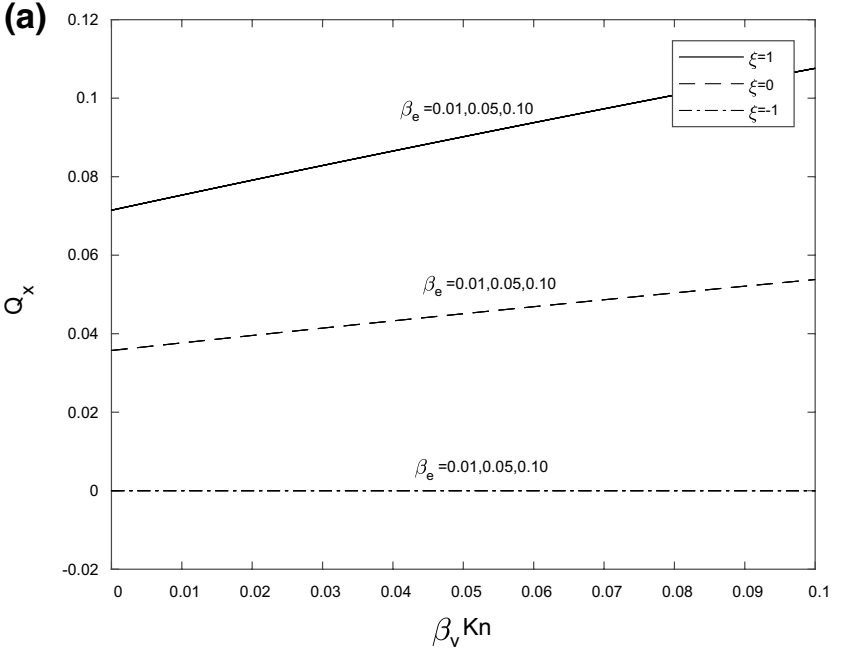

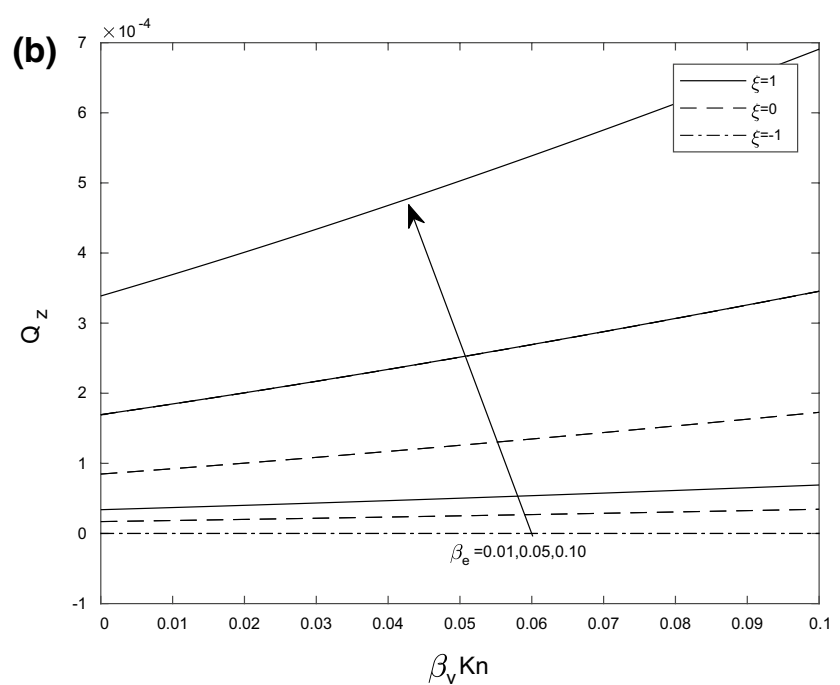

Fig. 7 Effect of Hall current parameter $\left(\beta_{\mathrm{e}}\right)$ on primary and secondary volume flow rate for $M=5.0, \beta_{\mathrm{i}}=3.0$ and $\ln =1.667$

for asymmetric heating $(\xi=-1)$ net volume flow is almost zero for all considered values of $M$ and $\beta_{\mathrm{v}} K n$.

Volume flow rate variations with Hall current parameter $\left(\beta_{\mathrm{e}}\right)$ and rarefaction parameter $\left(\beta_{\mathrm{v}} K n\right)$ are illustrated in Fig. 7 under different micro-channel heating conditions. It is be observed that as Hall parameter $\left(\beta_{\mathrm{e}}\right)$ increases, the volume flow along the primary flow direction remains unaffected for all considered values of $\xi$. This result is in excellent agreement with Fig. 3a as observed earlier. Increase in rarefaction parameter on the other hand yields an increase in volume flow for both symmetric ( $\xi=1)$ and asymmetric $(\xi=0)$ heating conditions. For secondary volume flow rate, it is observed that simultaneously increasing $\beta_{\mathrm{v}} K n$ and $\beta_{\mathrm{e}}$ causes an enhancement in volume flow rate for $\xi=1$ and $\xi=0$.

Figure 8 a shows that ion-slip parameter $\left(\beta_{\mathrm{i}}\right)$ supports flow formation for all considered values of $\beta_{v} K n$ when the micro-channel walls are heated either symmetrically $(\xi=1)$ or asymmetrically $(\xi=0)$ yielding an increase in primary volume flow rate $\left(Q_{x}\right)$, whereas the reverse is encountered for secondary volume flow rate $\left(Q_{z}\right)$.

Table 1 demonstrates the effects of Hartmann number $(M)$, Hall parameter $\left(\beta_{\mathrm{e}}\right)$ and ion-slip parameter $\left(\beta_{\mathrm{i}}\right)$ on skin friction in both primary and secondary flow directions $\left(\tau_{x}, \tau_{z}\right)$ on micro-channel walls situated at $y=0$ and $y=1$, respectively, when $\beta_{\mathrm{v}} K n=0.05, \ln =1.667$ and $\xi=0$. 

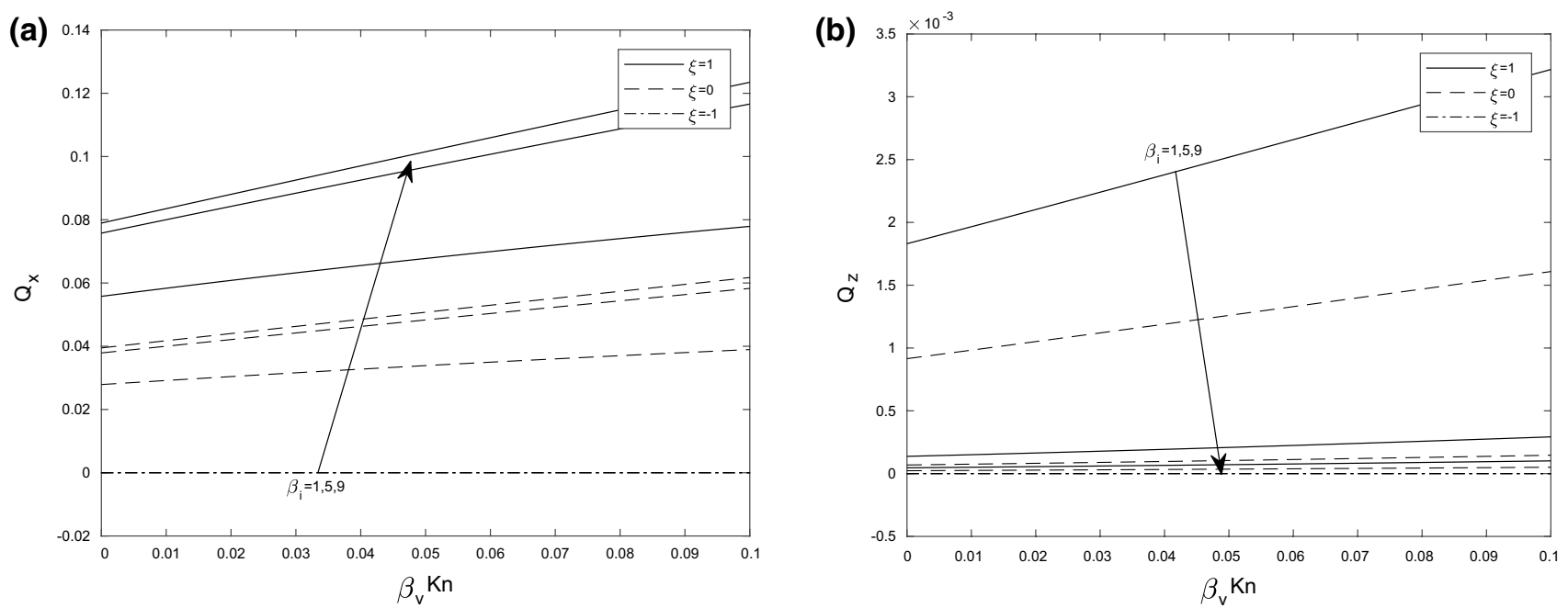

Fig. 8 Effect of ion-slip parameter $\left(\beta_{\mathrm{i}}\right)$ on primary and secondary velocity profiles for $\beta_{\mathrm{e}}=0.10, M=5.0$ and $\ln =1.667$

Table 1 Effects of Hartmann number $(M)$, Hall parameter $\left(\beta_{\mathrm{e}}\right)$ and ion-slip parameter $\left(\beta_{\mathrm{i}}\right)$ on skin friction on micro-channel walls situated at $y=0$ and $y=1$. For $\beta_{\mathrm{v}} K n=0.05, \mathrm{In}=1.667$ and $\xi=0$

\begin{tabular}{|c|c|c|c|c|c|c|}
\hline M & $\beta_{\mathrm{e}}$ & $\beta_{\mathrm{i}}$ & $\tau_{x 0}$ & $\tau_{z 0}$ & $\tau_{x 1}$ & $\tau_{z 1}$ \\
\hline \multirow[t]{9}{*}{5} & \multirow[t]{3}{*}{0.01} & 1 & 0.1074 & $4.6888 e-04$ & -0.2236 & $-5.8902 \mathrm{e}-04$ \\
\hline & & 5 & 0.1624 & $4.0136 \mathrm{e}-05$ & -2.2892 & $-4.6082 e-05$ \\
\hline & & 9 & 0.1718 & $1.3737 e-05$ & -0.3000 & $-1.5619 e-05$ \\
\hline & \multirow[t]{3}{*}{0.05} & 1 & 0.1075 & 0.0023 & -2.2237 & -0.0029 \\
\hline & & 5 & 0.1624 & $2.0067 e-04$ & -0.2892 & $-2.3039 e-04$ \\
\hline & & 9 & 0.1718 & $6.8682 \mathrm{e}-05$ & -0.3000 & $-7.8092 e-05$ \\
\hline & \multirow[t]{3}{*}{0.10} & 1 & 0.1077 & 0.0047 & -0.2239 & -0.0059 \\
\hline & & 5 & 0.1624 & $4.0124 \mathrm{e}-04$ & -0.2893 & $-4.6067 e-04$ \\
\hline & & 9 & 0.1718 & $1.3735 \mathrm{e}-04$ & -0.3000 & $-1.5617 e-04$ \\
\hline \multirow[t]{9}{*}{8} & \multirow[t]{3}{*}{0.01} & 1 & 0.0848 & $4.8431 \mathrm{e}-04$ & -0.1942 & $-6.5069 e-04$ \\
\hline & & 5 & 0.1512 & $5.6265 e-05$ & -0.2762 & $-6.5463 e-05$ \\
\hline & & 9 & 0.1647 & $2.0330 \mathrm{e}-05$ & -0.2918 & $-2.3285 e-05$ \\
\hline & \multirow[t]{3}{*}{0.05} & 1 & 0.0849 & 0.0024 & -0.1943 & -0.0033 \\
\hline & & 5 & 0.1512 & $2.8131 \mathrm{e}-04$ & -0.2762 & $-3.2729 e-04$ \\
\hline & & 9 & 0.1647 & $1.0165 \mathrm{e}-04$ & -0.2918 & $-1.1642 e-04$ \\
\hline & \multirow[t]{3}{*}{0.10} & 1 & 0.0850 & 0.0048 & -0.1946 & -0.0065 \\
\hline & & 5 & 0.1512 & $5.6250 e-04$ & -0.2762 & $-6.5445 e-04$ \\
\hline & & 9 & 0.1647 & $2.0328 \mathrm{e}-04$ & -0.2918 & $-2.3282 \mathrm{e}-04$ \\
\hline \multirow[t]{9}{*}{10} & \multirow[t]{3}{*}{0.01} & 1 & 0.0741 & $4.7111 \mathrm{e}-04$ & -0.1796 & $-6.6117 e-04$ \\
\hline & & 5 & 0.1444 & $6.4670 \mathrm{e}-05$ & -0.2683 & $-7.5918 \mathrm{e}-05$ \\
\hline & & 9 & 0.1602 & $2.4162 \mathrm{e}-05$ & -0.2867 & $-2.7809 e-05$ \\
\hline & \multirow[t]{3}{*}{0.05} & 1 & 0.0742 & 0.0024 & -0.1796 & -0.0033 \\
\hline & & 5 & 0.1444 & $3.2333 e-04$ & -0.2683 & $-3.7956 e-04$ \\
\hline & & 9 & 0.1602 & $1.2080 \mathrm{e}-04$ & -0.2867 & $-1.3904 \mathrm{e}-04$ \\
\hline & \multirow[t]{3}{*}{0.10} & 1 & 0.0743 & 0.0047 & -0.1799 & -0.0066 \\
\hline & & 5 & 0.1444 & 6.4654 & -0.2683 & $-7.5898 \mathrm{e}-04$ \\
\hline & & 9 & 0.1602 & $2.4159 e-04$ & -0.2867 & $-2.7806 e-04$ \\
\hline
\end{tabular}

Details from the table reveals that in the presence of Hall current $\left(\beta_{\mathrm{e}}\right)$ and ion slip $\left(\alpha_{\mathrm{e}}\right)$, increase in Hartmann number $(M)$ causes a reduction in $\tau_{x 0}$, whereas it increases $\tau_{x 1}$.
Increase in ion slip $\left(\beta_{\mathrm{i}}\right)$, however, decreases $\tau_{x 0}$ while it displays a dual character for $\tau_{x 1}$. For the secondary component, on the other hand $\left(\tau_{z}\right)$, evidence from the table 
shows that $\tau_{z 0}$ increases with Hall parameter whereas it decreases $\tau_{z 1}$. Increase in Hartmann number causes an increase in $\tau_{z 0}$ while it displays a dual character for $\tau_{z 1}$.

Numerical results for fluid velocity obtained in this work are compared with those of Jha et al. [21] in the absence of ion-slip current and presented in Table 2. Details from the table suggest that in the absence of ion-slip current $\left(\beta_{\mathrm{i}}=0.0\right)$, numerical values of present work are in excellent agreement with those of Jha et al. [21], hence validating the solution obtained herein. Also as a special case, numerical values for fluid velocity obtained in the present work are validated with those of Chen and Weng [41] and presented in Table 3 in the absence of a transversely applied magnetic field, i.e., $M \rightarrow 0$.

\section{Conclusion}

Combined effect of Hall and ion-slip current on hydromagnetic free convection flow of viscous incompressible and electrically conducting fluid in a vertical micro-channel is investigated taking into consideration the asymmetric heating of the micro-channel walls. Detail from the analysis leads to the resulting conclusions:

1. In the presence of ion-slip current, primary fluid velocity and volume flow remains unaffected with variations in Hall current parameter for all micro-channel heating conditions.

2. Increase in Hartmann number decreases fluid velocity along the primary direction, whereas it enhances it along the secondary flow direction.

3. In the presence of ion-slip current, secondary volume flow rate could be enhanced by simultaneously
Table 3 Numerical comparison of the values of the primary fluid velocity obtained in the present work (for $M \rightarrow 0$, i.e., in the absence of Hall and ion-slip current) with those of Chen and Weng [41]. When $\mathrm{In}=1.667$ and $\beta_{\mathrm{v}} K n=0.05$

\begin{tabular}{lllc}
\hline$\xi$ & $y$ & Chen and Weng [41] & $\begin{array}{c}\text { Present work } \\
(\text { When } M \rightarrow 0)\end{array}$ \\
\hline 1.0 & 0.0 & 0.0250 & 0.0250 \\
& 0.2 & 0.1050 & 0.1050 \\
& 0.4 & 0.1450 & 0.1450 \\
& 0.6 & 0.1450 & 0.1450 \\
& 0.8 & 0.1050 & 0.1050 \\
0.0 & 1.0 & 0.0250 & 0.0250 \\
& 0.0 & 0.0157 & 0.0157 \\
& 0.2 & 0.0437 & 0.0437 \\
& 0.4 & 0.0684 & 0.0684 \\
& 0.6 & 0.0766 & 0.0766 \\
& 0.8 & 0.0613 & 0.0613 \\
& 1.0 & 0.0157 & 0.0157 \\
-1.0 & 0.0 & -0.0065 & -0.0065 \\
& 0.2 & -0.0176 & -0.0176 \\
& 0.4 & -0.0081 & -0.0081 \\
& 0.6 & 0.0081 & 0.0081 \\
& 0.8 & 0.0176 & 0.0176 \\
& 1.0 & 0.0065 & 0.0065 \\
\hline
\end{tabular}

increasing the rarefaction parameter and Hall current parameter.

4. For asymmetric heating $(\xi=0)$, skin friction for the primary flow direction at $y=0$ could be reduced with the increase in Hartmann number.

Table 2 Numerical comparison for fluid velocity in the present work with those of Jha et al. [21] in the absence of ion-slip current $\left(\beta_{\mathrm{i}}=0.0\right)$, when $\beta_{\mathrm{v}} K n=0.05, \mathrm{In}=1.667, M=5$ and $\beta_{\mathrm{e}}=0.5$

\begin{tabular}{|c|c|c|c|c|c|}
\hline$\xi$ & $y$ & $\begin{array}{l}u \\
\text { Present work }\end{array}$ & $\begin{array}{l}w \\
\text { Present work }\end{array}$ & $\begin{array}{l}u \\
\text { Jha et al. [21] }\end{array}$ & $\begin{array}{l}\text { w } \\
\text { Jha et al. [21] }\end{array}$ \\
\hline \multirow{4}{*}{1.0} & 0.2 & 0.0679 & 0.0110 & 0.0679 & 0.0110 \\
\hline & 0.4 & 0.0938 & 0.0158 & 0.0938 & 0.0158 \\
\hline & 0.6 & 0.0938 & 0.0158 & 0.0938 & 0.0158 \\
\hline & 0.8 & 0.0697 & 0.0110 & 0.0697 & 0.0110 \\
\hline \multirow[t]{4}{*}{0.0} & 0.2 & 0.0270 & 0.0051 & 0.0270 & 0.0051 \\
\hline & 0.4 & 0.0433 & 0.0077 & 0.0433 & 0.0077 \\
\hline & 0.6 & 0.0505 & 0.0081 & 0.0505 & 0.0081 \\
\hline & 0.8 & 0.0427 & 0.0059 & 0.0427 & 0.0059 \\
\hline \multirow[t]{4}{*}{-1.0} & 0.2 & -0.0156 & $-0.8508 \mathrm{e}-03$ & -0.0156 & $-0.8508 \mathrm{e}-03$ \\
\hline & 0.4 & -0.0071 & $-0.4339 e-03$ & -0.0071 & $-0.4339 e-03$ \\
\hline & 0.6 & 0.0071 & $0.4339 e-03$ & 0.0071 & $0.4339 e-03$ \\
\hline & 0.8 & 0.0159 & $0.8508 e-03$ & 0.0159 & $0.8508 \mathrm{e}-03$ \\
\hline
\end{tabular}




\section{Compliance with ethical standards}

Conflict of interest The authors declare that they have no conflict of interest.

\section{Appendix}

$$
\begin{aligned}
& C_{1}=\xi+\frac{\beta_{\mathrm{v}} K n(1-\xi)}{1+2 \beta_{\mathrm{v}} K n \ln } \\
& C_{2}=\frac{1-\xi}{1+2 \beta_{\mathrm{v}} K n \ln }
\end{aligned}
$$

$k_{1}=\beta_{\mathrm{v}} K n M_{1}$

$k_{2}=\frac{1}{M_{1}^{2}\left(A_{1} \beta_{v} K n-A_{0}\right)}$

$k_{3}=\cosh \left(M_{1}\right)+M_{1} \beta_{\mathrm{v}} K n \sinh \left(M_{1}\right)$

$k_{4}=\sinh \left(M_{1}\right)+M_{1} \beta_{v} K n \cosh \left(M_{1}\right)$

$k_{5}=-\frac{\left(\left(C_{1}+C_{2}\right)+C_{2} \beta_{v} K n\right)}{M_{1}^{2}}$

$C_{3}=\frac{k_{1} k_{5}+k_{2} k_{4}}{k_{4}+k_{1} k_{3}}$

$C_{4}=\frac{k_{5}-k_{2} k_{3}}{k_{4}+k_{1} k_{3}}$

\section{References}

1. Krishan V (1999) Magnetohydrodynamics of conducting fluids, astrophysical plasmas and fluids. Astrophysics and Space Science Library, vol. 235. Springer, Dordrecht, pp 117-195

2. Chiolerio A, Quadrelli MB (2017) Smart fluid systems: the advent of autonomous liquid robotics. Adv Sci 4(7):1-18

3. Chiolerio A, Quadrelli MB (2018) Colloidal energetic systems. Energy Technol 7(5):1-31

4. Jha BK, Babatunde A, Joseph SB (2014) Natural convection flow in a vertical micro-channel with suction/injection. J Process Mech Eng 228(3):171-180

5. Jha BK, Aina B (2017) Magnetohydrodynamic mixed convection flow in vertical microchannel in the presence of induced magnetic field. Int J Appl Mech Eng 22(3):567-582

6. Jha BK, Aina B (2017) Impact of induced magnetic field on MHD mixed convection flow in vertical micro-channel formed by nonconducting and conducting infinite vertical parallel plates. J Nanofluids 6(5):960-970

7. Jha BK, Aina B (2016) Role of induced magnetic field on MHD natural convection flow in a vertical microchannel formed by two electrically non-conducting infinite vertical parallel plates. Alexandria Eng J (Faculty of Engineering, Alexandria University) 55(3):2087-2097

8. Aydin O, Avci M (2006) Heat and fluid flow characteristics of gas in micro-pipes. Int J Heat Transf 49(9-10):1723-1730
9. Avci M, Aydin O (2007) Mixed convection in a vertical parallel plate micro-channel. J Heat Transf Trans ASME 129(12):162-166

10. Buonomo B, Manca O (2012) Transient natural convection flow in a vertical micro-channel with heated at uniform heat flux. Int J Therm Sci 56(2):35-47

11. Hossain MA, Mandal AC (1985) Mass transfer effects on the unsteady hydromagnetic free convection flow past an accelerated vertical porous plate. J Phys D Appl Phys 18:L63-L69

12. Ibrahim FS, Hassanien IA, Bakr AA (2004) Unsteady magnetohydrodynamic micro-polar fluid flow and heat transfer over a vertical porous plate through a porous medium in the presence of thermal and mass diffusion with a constant heat source. Can J Phys 82:775-790

13. Eldabe NTM, Elbashbeshy EMA, Hasanin WSA, Elsaid EM (2011) Unsteady motion of MHD viscous incompressible fluid with heat and mass transfer through porous medium near a moving vertical plate. Int J Energy Technol 3:1-11

14. Gorla RSR, Chamkha AJ (2011) Natural convective boundary layer flow over a horizontal plate embedded in a porous medium saturated with a nanofluid. J Mod Phys 2:62-71

15. Cramer KR, Pai SI (1973) Magnetofluid-hydrodynamics for engineers and applied physicist. McGraw-Hill Book Comp, New York

16. Sato $H$ (1961) The Hall effect in the viscous flow of ionized gas between parallel plates under transverse magnetic field. J Phys Soc Jpn 16:1427-1433

17. Datta N, Jana RN (1975) Hall effect on free convection between vertical parallel plates. Meccanica 10(4):239-245

18. Data N, Jana RN (1977) Hall effects on hydromagnetic convective flow through a channel with conducting walls. Int J Eng Sci 15:561-567

19. Mazumder BS, Gupta AS, Datta N (1976) Flow and heat transfer in the hydromagnetic Ekman layer on a porous plate with Hall effects. Int J Heat Transf 19:523-527

20. Guchhait S, Das S, Jana RN, Ghosh SK (2011) combine effects of Hall current and rotation on unsteady Couette flow in a porous channel. World J Mech 1:87-99

21. Jha Basant K, Malgwi Peter B, Aina Babatunde (2018) Hall effect on MHD natural convection flow in a vertical micro-channel. Alexandria Eng J. https://doi.org/10.1016/j.aej.2017.01.038

22. Singh AK (1984) Hall effect on MHD Free-convection flow past an accelerated vertical porous plate. Astrophys Space Sci 102:213-221

23. Seth GS, Ansari Md (2009) Magnetohydrodynamics convective flow in a rotation channel with Hall effect. Int JTheor Appl Mech 4(2):205-222

24. Seth GS, Singh JK (2015) Mixed convection hydromagnetic flow in a rotating channel with Hall and wall conductance effects. J Appl Math Model Elsevier 40:2783-2803

25. Makinde OD, Iskander T, Mabood F, Khan WA, Tshehla MS (2016) MHD "Coquette-poiseuille flow of variable viscosity nanofluid in a roating permeable channel with Hall effects". J Mol Liq 221:778-787

26. Krishna VM, Jyothi K (2018) Hall effects on MHD rotating flow of a visco-elastic fluid through a porous medium over an infinite oscillating porous plate with heat source and chemical reaction. Mater Today Proc 5:367-380

27. Tani I (1962) Steady flow of conducting fluids in channels under transverse magnetic fields with consideration of Hall effects. J Aerosp Sci 29:297-305

28. Mittal ML, Masapathi GH, Rao BN (1976) Entrance flow in a MHD channel with Hall and ion-slip currents. AIAA J 14(12):17681770. https://doi.org/10.2514/3.7285

29. Ram PC, Singh AK, Takhar HS (1995) Effects of Hall and ion-slip currents on convective flow in a rotating fluid with a wall temperature oscillation. Magnetohydrodyn Plasma Res 5:1-16 
30. Jha BK, Clement AA (2010) Combined effect of Hall and ion-slip currents on unsteady MHD Couette flow in a rotation system. J Phys Soc Jpn 79:104401

31. Naroua H, Takhar HS, Ram PC, Beg TA, Beg OA, Bhargava R (2007) Transient rotating hydromagnetic partially-ionized heat-generating gas dynamic flow with Hall/ionslip current effects: finite element analysis. Int J Fluid Mech Res 34(6):493-505. https:// doi.org/10.1615/InterJFluidMechRes.v34.i6.10

32. Singh JK, Naveen J, Ghousia B (2016) Unsteady Magnetohydrodynamic Couette-Poiseuille flow within porous plates filled with Porous medium in the presence of a moving magnetic field with Hall and ion-slip effects. Int J Heat Technol 34:89-97

33. Srinivasacharya D, Shafeeurrahman M (2017) Hall and ion slip effects on MHD mixed convection flow of nanofluid between two concentric cylinders. J Assoc Arab Univ Basic Appl Sci. http://dx.doi.org/10.1016/j.jaubas.2017.03.002

34. Attia HA, Aboul-Hassan AL (2003) The effect of variable properties on the un-steady Hartmann flow with heat transfer considering the Hall effect. Appl Math Model. 27:551-563

35. Singh JK, Begum SG, Joshi N (2006) Effects of Hall current and ion-slip on unsteady hydromagnetic generalized Couette flow in a rotating darcian channel. J Math Model 3(2):145-167
36. Makinde OD, Iskander T, Mabood F, Khan WA, Tshehla MS (2016) MHD Couette-Poiseuille flow of variable viscosity nano-fluids in a rotating permeable channel with Hall effects. J Mol Liq 221:778-787

37. Ghosh SK, Anwar Bég O, Zueco J (2010) Hydromagnetic free convection flow with induced magnetic field effects. Mecanica 45:175-185

38. Mollah MdT, Islam MM, Alam MdM (2018) Hall and ion-slip effects on unsteady MHD Bingham fluid flow with suction. Model Meas Control B 87(4):221-229

39. Eckert ERG, Drake RM Jr (1972) Analysis of heat and mass transfer. Mcgraw-Hill, New York, Chap, p 11

40. Goniak R, Duffa G (1995) Corrective term in wall slip equations for Knudsen layer. J Thermophys Heat Transf 9:38-383

41. Chen CK, Weng HC (2005) Natural convection in a vertical microchannel. J Heat Transf 127:1053-1056

42. Weng HC, Chen DC (2017) Magnetogasdynamics natural convection in a long vertical micro-channel. Appl Math Model. https ://doi.org/10.1016/j.apm.2017.02.007

Publisher's Note Springer Nature remains neutral with regard to jurisdictional claims in published maps and institutional affiliations. 